\title{
Comparison of Dynamic Balance in Football Players with and without Genu Varum Using Star Excursion Balance Test
}

\author{
Nirbhay Shah ${ }^{1}$, Anagha Palkar ${ }^{2}$ \\ ${ }^{1}$ Student (BPT) DPO's Nett College of Physiotherapy, Thane \\ ${ }^{2}$ Assistant Professor, DPO's Nett College of Physiotherapy, Thane
}

Corresponding Author: Nirbhay Shah

\begin{abstract}
Background and Aims: Genu Varum is an angular deformity of the knee that is responsible for the alteration of the forces at the knee so that the line of force shifts farther medially from the knee joint centre intensifying the medial compartment load and creating a medial joint reaction force that is nearly three and a half times that of the lateral compartment. Football is a high intensity sport that places inordinate amounts of load and torque on the knee joint making it more susceptible to growth deformities in the developing age groups. Genu varum is a predisposing factor to lateral instability which affects dynamic balance. This study consists of a comparison in dynamic balance between professional football players, those without a knee deformity and those with genu varum deformity using the Star excursion balance test.

Methodology: A comparative study was conducted among 40 elite level football players and they were divided into two groups one of which consisted football players without a knee deformity and the other group which comprised of football players with genu varum. Genu varum was calculated using the intercondylar distance measure. Star excursion balance test was used to assess dynamic balance of each individual and their average distances were calculated for each direction in the test.

Results: The Reach Distances in the anterior direction showed no significant difference $(\mathrm{P}>0.05)$ between the control and Group Bs whereas significant differences $(\mathrm{P}<0.05)$ were obtained in the Posteromedial and Posterolateral directions

Conclusion: There was no significant difference in reach distances in the anterior direction but significant differences in the reach distances were observed in the posteromedial as well as the posterolateral direction suggesting that genu varum deformity does affect the dynamic balance in elite level football players.
\end{abstract}

Keywords: Genu Varum, Football Players, Dynamic Balance, Star excursion balance test

\section{INTRODUCTION}

Football is the most popular sport in the world and is performed by men and women, children and adults with different levels of expertise. ${ }^{[2]}$ Football performance depends upon a myriad of factors such as technical/biomechanical, tactical, mental and physiological areas. It is one of the most commonly played sport at both elite and grassroots level in India. Playing football regularly exposes the knee joint to load and torque stresses that contribute to genu varum in the lower limbs

The load-bearing axis of the lower limb can be represented by a line extending from femoral head centre to ankle joint centre. In a Genu varus (bow-leg) knee, this line passes medial to the centre of the knee, increasing force across the medial tibiofemoral compartment. Genu varum is one of the angular deformities of the knee. ${ }^{[1]}$ It is one of the most common anatomic 
variations of musculoskeletal alignment. Lower limb deformities such as genu varum are prevalent in children. ${ }^{[1]}$ It is caused by numerous factors including developmental bowing, congenital bowing, tibia vara (Blount's disease), neurofibromatosis, rickets, osteogenesis imperfecta, camptomelic dysplasia, achondroplasia, and even intense exercise. It is measured by the Intercondylar (IC) distance between both the knee joints when a person is standing with their feet close to each other. A distance of up to $6 \mathrm{~cm}$ is considered as a normal value. ${ }^{[1]}$

In Football, the knee joint sustains an inordinate amount of load and torque, particularly when sustained at a higher level of competition. High amount of stress and strain imposed on a joint during growth and adolescence through intensive practice of a sport may contribute to a growth deformity. In addition to the development of tibiofemoral osteoarthritis, the presence of genu varum seems also to predispose subjects to the occurrence of injuries at the patellofemoral joint. The presence of genu varum alters the forces at the knee so that the line of force shifts farther medially from the knee joint centre intensifying the medial compartment load and creating a medial joint reaction force that is nearly three and a half times that of the lateral compartment.

Balance is a physiological and mechanical situation and the desire of moving body within the optimal level of support. It can be grouped into static and dynamic categories. Static balance is the ability to maintain postural stability and orientation with center of mass (COM) over base of support (BOS) and the body at rest. Dynamic balance is the ability to maintain postural stability and orientation with $\mathrm{COM}$ over BOS while body is in motion. ${ }^{[8]}$ The maintenance and control of balance, whether under static or dynamic conditions, is an essential requirement for physical and daily activities.

The SEBT can be used to provide objective measures to differentiate deficits and improvements in dynamic balance related to lower extremity injury and induced fatigue, and it has the potential to predict lower extremity injury. ${ }^{7]}$ It is an important clinical functional performance test requiring adequate range of motion, muscle strength and proprioceptive and neuromuscular adjustments to keep balance. $^{[7]}$ It is being used to identify postural and balance control deficiencies related to lower-limb pathology, detect improvements in balance rehabilitation, predict lower limb injury and train both patients and healthy people. However, it can be time-consuming when evaluating or rehabilitating a patient in the clinic with the recommended number of repetitions in each of the eight directions of the SEBT. Hence, in the last few years, several authors have used three specific directions (Anterior, Posteromedial, and Posterolateral) of the SEBT because it was reported that these three directions measure the same constructs as other five directions of the SEBT..$^{[7,8]}$

Since genu varum predisposes to knee injuries, and many children participate in soccer at a high-level during youth, there is a great need to scientifically investigate the relationship between genu varum and soccer participation.

Approximately 3-11\% of children sustain injuries while participating in various sports, especially soccer. Playing soccer regularly exposes the knee joint to load and torque stresses that contribute to genu varum in the lower limbs ${ }^{[1,2]}$

Shojaedin et al., (2012) reported that subjects with genu varum deformity revealed higher peak of the vertical ground reaction force in calcareous contact in comparison of subjects with normal knee

Besier et al. measured the external loading of the knee joint during Cut and plant movements and concluded that varus and valgus stress on the knee joint was dramatically increased during these tasks. Hence football players are more prone to knee injuries.

Akram Pashnameh et al. studied the relationship between genu valgum, genu varum and flat foot Deformities with Static 
and Dynamic Balance in Female Students and concluded that genu varum was the only deformity that had an effect on the static and dynamic balance. ${ }^{[6]}$ These results support the hypothesis that a high amount of stress and strain imposed on a joint during growth through intensive practice of a sport may contribute to a growth deformity

Due paucity of literature comparing the prevalence of Genu varum with impaired balance in football players this study must be undertaken

\section{METHODOLOGY}

Study Design- Comparative Study

Sample Design

Sample Population- Male Football Players Age $=18$-30years

Sample size-40

Sampling type- Convenient sampling

\section{- Inclusion criteria}

1. Players willing to participate for the study

2. Age group-18-30years

3. Players who have undergone training for 3 years or more

4. Players having Genu Varum deformity

5. Players without genu varum deformity

6. Players without history of long-term injuries

7. Football players who have played district level or more

\section{- Exclusion Criteria}

1. Players With any injuries in the last 6 months

2. Players with congenital lower limb deformities

3. Players who have undergone surgery in the past 3 years

4. Players undergoing any form of physiotherapy treatment for lower limb injuries

Ethical Clearance: The Study was reviewed by the ethical committee

Permission to conduct the Study was taken from the football club. A written informed consent was taken from all the subjects prior to the study. All Covid-19 related precautions were taken and screening will be done for the same. 40 competitive Football players who were willing to participate in the study were included. All the study subjects were screened as per the inclusion and the exclusion criteria. The subjects were explained about the procedure and the purpose of this study. They were divided in two groups- Football players without genu varum (Group A) and Football players with Genu varum (Group B). That was determined by measuring the intercondylar (IC) distance. This was done by asking the subject to take a relaxed erect standing position on a platform with the feet at shoulder width, each foot on a different platform The subjects were instructed to position their feet in such a manner that the medial border of the feet was above the inner border of the platform. The intercondylar distance of up to $3 \mathrm{~cm}$ is considered normal and those with distances of $4 \mathrm{~cm}$ or greater were grouped under the Group B

\section{To Assess Dynamic Balance using SEBT}

$4-120 \mathrm{~cm}$ lengths of sticky tape were applied on to the floor, intersecting in the middle, and with the lines placed at $45^{\mathrm{O}}$ angles, Making a Star. The SEBT was originally designed as a lower extremity reach test performed on an 8-directions The test has been suggested to be simplified to include just 3 directions, such as anterior (ANT), posterolateral (PL), and posteromedial (PM). The subjects were then required to stand in the center of the star with their hands firmly placed on their hips, they were then instructed to reach with one foot as far as possible and lightly touch the line before returning back to the starting upright position. The spot at which the athlete touched his toe was then marked and recorded. Average distance in each direction $(\mathbf{c m})=$ Reach $1+$ Reach $2+$ Reach 3 / 3. Average distances were calculated and the data was then analyzed. 
Nirbhay Shah et.al. Comparison of dynamic balance in football players with and without genu varum using star excursion balance test.

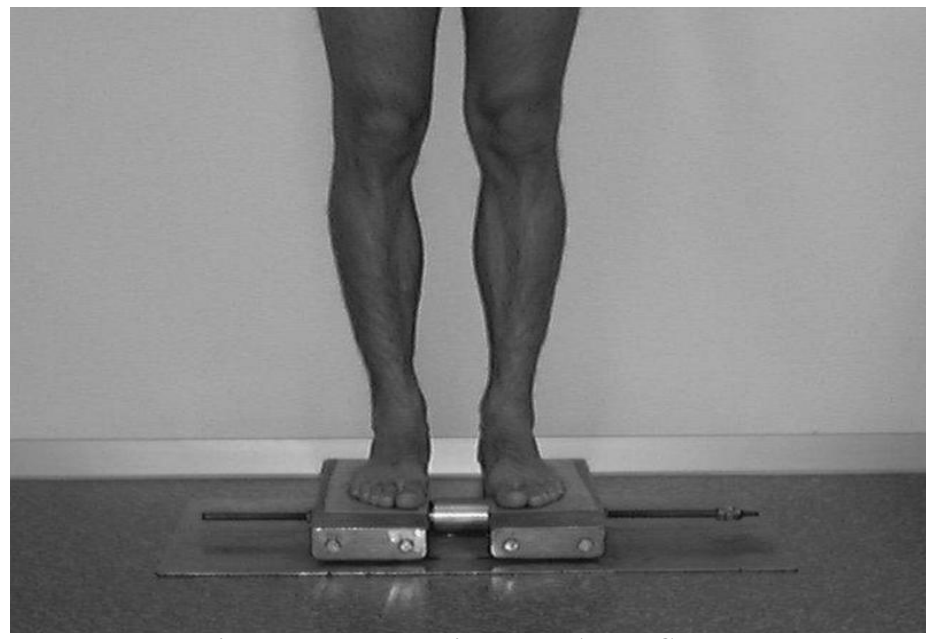

Measuring Intercondylar Distance to Assess Genu Varum

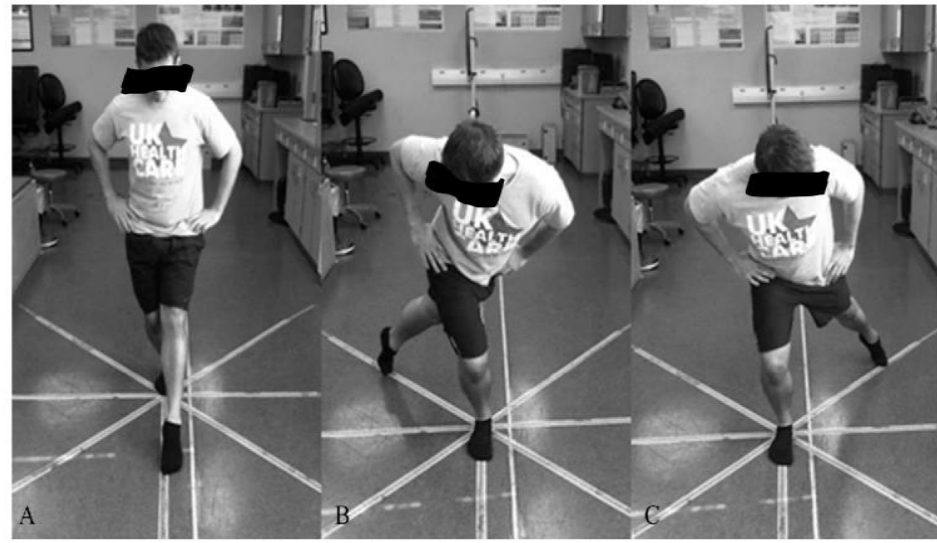

Performing Star excursion Balance test. A- Anterior Direction, B- Posteromedial Direction C- Posterolateral Direction

\section{DATA ANALYSIS AND RESULTS}

The Data collected was then analyzed using GraphPad Prism 9. Normality of the data was checked using the Shapiro-Wilk Test. The Man Whitney's Test was used to compare the reach distances in the anterior direction on both control and Group B. The unpaired-T test was used to compare data on the posteromedial and posterolateral direction between the control and Group Bs.
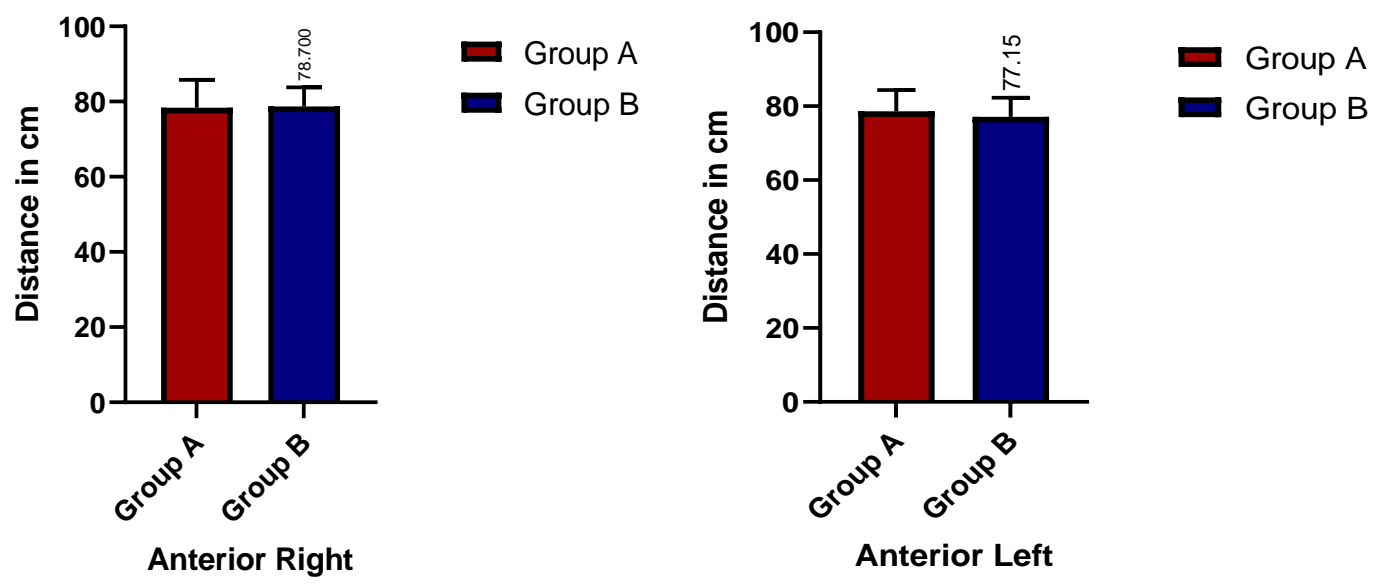

Graph 1(Right and Left Legs) 


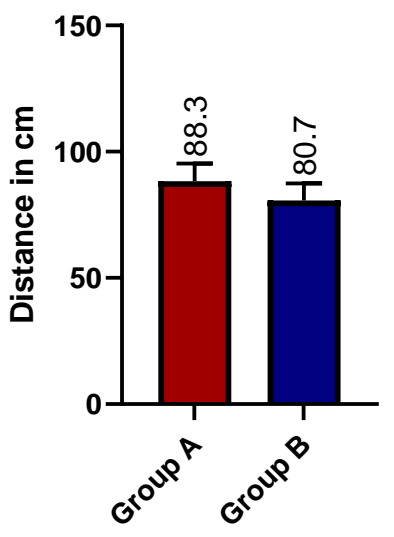

Right Posterolateral

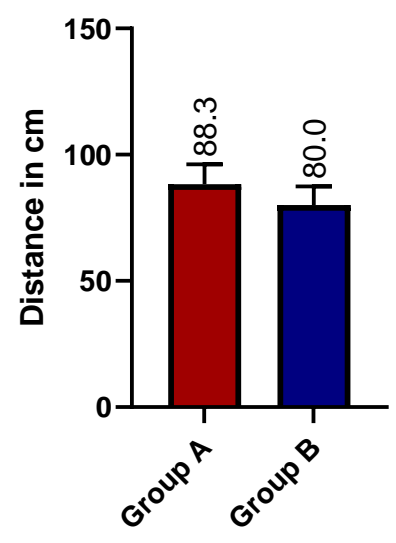

Left Posterolateral
Group A
Group B

Group B

Graph 2(Right and Left Legs)
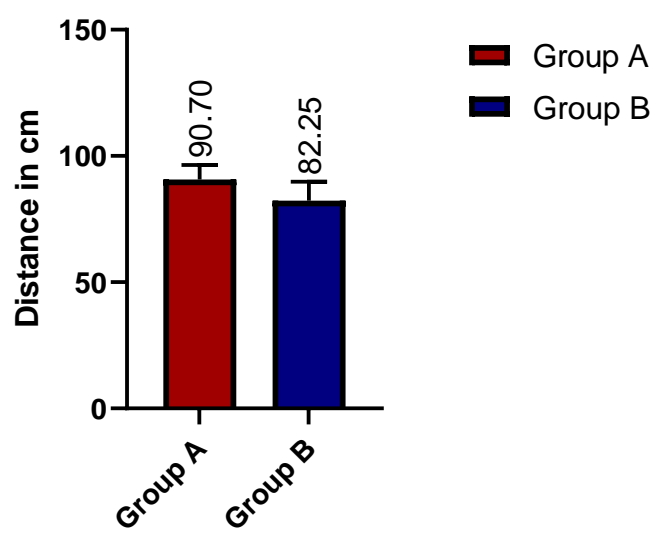

Right Posteromedial

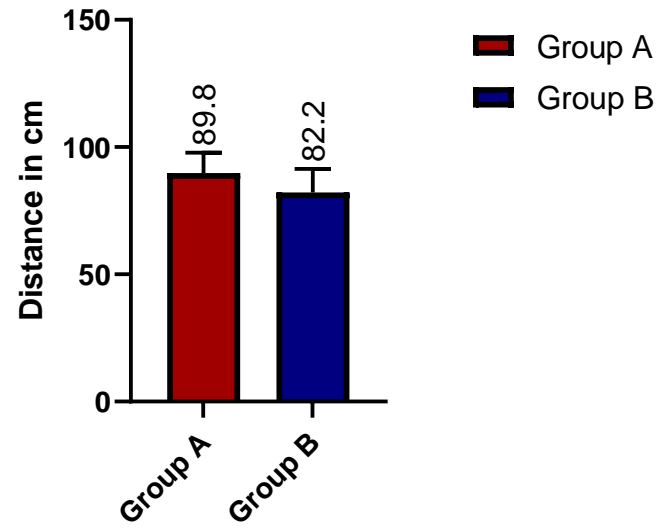

Left Posteromedial

Graph 3 (Right and left legs)

\section{RESULTS}

The Reach Distances in the anterior direction showed no significant difference $(\mathrm{P}>0.05)$ between the control and Group Bs whereas significant differences $(\mathrm{P}<0.05)$ were obtained in the Posteromedial and Posterolateral directions

\section{DISCUSSION}

Football (soccer) is the most commonly played sport at both elite and grassroots level in many countries. ${ }^{[2]} \mathrm{A}$ survey found that football is by far the most popular sport among male children and adolescents. Alignment in the knee joint is a key indicator of the load distribution, and asymmetry of weight bearing is a cause to threat postural control. Genu varum deformity can cause gait problems, chronic fatigue, spinal deformity, aging, back pain, and neuralgia. It also affects the risk of medial osteoarthritis disease progression, because imbalanced medial transmission of weight increases the medial compression force on the medial compartment of the knee joint. ${ }^{[1,2,5]}$ It decreases functional status, and increases the risk of injuries, falls, or collision. the SEBT is a series of single-limb squats using the non-stance limb to reach maximally to touch a point along 1 of 8 designated lines on the ground. However, in the last few years, several authors have used three specific directions (Anterior, Posteromedial, and Posterolateral) of the SEBT because it was reported that these three directions measure the same constructs as other five directions of the SEBT. ${ }^{[5,7,8]}$

This study was conducted to compare the SEBT values in 40 elite Football players, those with and without Genu varum in order to come to a 
conclusion as to whether genu varum does affect the overall dynamic balance and hence sports performance in highly trained football players.

As mentioned above, 3 reach directions were considered while using the SEBT (Anterior, Posteromedial, and Posterolateral). Results were calculated by comparing the two groups. The Group A was football players without Genu Varum $(n=20)$ and the Group B was football players with Genu varum $(n=20)$.

\section{Anterior Direction}

For both the stance limbs (Right and left) the Anterior reach distances were calculated and compared using the Man Whitney test and results showed value to be $\mathrm{P}=0.9813$ on the right leg and significant difference was not found $(\mathrm{P}<0.05)$. The mean distance for the Group A was $78.50 \mathrm{~cm}$ and for the Group B was $79.50 \mathrm{~cm}$. Similar test on the left side $(\mathrm{P}=0.2382)$ showed no significant difference $(\mathrm{P}<0.05)$ with mean reach distances at 79.00 for the Group A and 77.50 for the Group B

Bhanot $\mathrm{K}$ et al. in their study stated that the EOB(External oblique) EMG activity was highest in the Anterior direction. It might be because of the trunk rotation to the opposite side of the stance leg to counterbalance the reaching leg. The reason for reach distances not having a significant difference could be because of genu varum having no significant effect in the trunk musculature and also that it is associated more with the lateral compartment weakness/ instability. ${ }^{[8]}$ Further research on this may help in finding conclusive results

\section{Posteromedial Direction}

On comparison of reach distances in the posteromedial direction on the right leg using the unpaired $\mathrm{T}$ test $(\mathrm{P}=0.003)$ showed significant difference $(\mathrm{P}<0.05)$ with mean value of $90.70 \mathrm{~cm}$ for the Group $A$ and $82.25 \mathrm{~cm}$ for the Group B. On the left leg, comparison of data using the unpaired $\mathrm{T}$ test $(\mathrm{P}=0.0082)$ again showed significant difference $(\mathrm{P}<0.05)$ and mean values of $89.90 \mathrm{~cm}$ on the Group A and $82.20 \mathrm{~cm}$ on the Group B.

\section{Posterolateral Direction}

On comparing the reach distances in the posterolateral direction on the right leg using the unpaired $\mathrm{T}$ test $(\mathrm{P}=0.0012)$ showed significant difference $(\mathrm{P}<0.05)$ with mean reach distances at $88.30 \mathrm{~cm}$ in the Group A and $80.70 \mathrm{~cm}$ in the Group B. Similar comparison on the left leg using the unpaired $\mathrm{T}$ test $(\mathrm{P}=0.0015)$ showed significant difference $(\mathrm{P}<0.05)$ with mean reach distances at $88.30 \mathrm{~cm}$ on the Group A and $80.0 \mathrm{~cm}$ in the Group B.

Yun-Won Chae, et al in their study The Effect of Postural Stability on Genu Varum in Young Adults suggested that Genu varum causes stretching of the posterolateral joint capsule and collateral ligament of the lower limb joints, and the weakness of the muscles that are involved in mediolateral stability, increase the instability of the lower limb joints. ${ }^{[9]}$ This may affect postural control and their study showed significant difference in MLSI (mediolateral stability index) between the Group A and genu varus group. This along with increased Gluteus medius firing on the EMG activity showed in studies suggests that due to weakness to the lateral compartment are most likely causes for the significant differences obtained in the reach directions between the two groups. ${ }^{[4,8,9]}$

\section{Limitations and Suggestions Limitations}

- Small Sample size

- Lack of training due to the pandemic

- Non-Availability of radiographs for accurate assessment of Knee deformities

\section{Suggestions}

- More studies can be carried out on a higher level of performing athletes

- Studies carried out in times with football players during the regular season

- A higher sample of players to be taken to get more accurate results 
- Measure Knee deformities using a radiographic evidence for more accurate assessment

- Use of EMG in order to objectively assess muscle activation

\section{CONCLUSION}

There was no significant difference in the reach distances in the anterior direction on both the stance legs between the football players with genu varum and the players without genu varum. However, Significant differences were seen in the posterolateral and posteromedial directions on both the stance legs with mean distances on the Group B being significantly lesser than the Group A to suggest that genu varum does affect the dynamic balance in football players with Genu Varum

\section{Acknowledgement: None}

\section{Conflict of Interest: None}

\section{Source of Funding: None}

\section{Ethical Approval: Approved}

\section{REFERENCES}

1. Asadi, K., Mirbolook, A., Heidarzadeh, A., Mardani Kivi, M., Emami Meybodi, M. and Rouhi Rad, M. (2015). Association of Soccer and Genu Varum in Adolescents. Trauma Monthly, 20(2).

2. Thaller, P., Fürmetz, J., Chen, F., Degen, N., Manz, K. and Wolf, F. (2018). Bowlegs and intensive football training in children and adolescents. Deutsches Aerzteblatt Online.

3. Mongashti Joni, Y., Fatahi, F., Ghanizadeh Hasar, N. and Hosseinpour, E. (2017). Effect of Genu Varum Deformity on Gluteus Medius Muscle Activity and Postural Control During Single-Leg JumpLanding. Physical Treatments: Specific Physical Therapy Journal, pp.79-88.
4. Samaei, A., Bakhtiary, A., Elham, F. and Rezasoltani, A. (2012). Effects of Genu Varum Deformity on Postural Stability. International Journal of Sports Medicine, 33(06), pp.469-473.

5. Gribble, P., Hertel, J. and Plisky, P. (2012). Using the Star Excursion Balance Test to Assess Dynamic Postural-Control Deficits and Outcomes in Lower Extremity Injury: A Literature and Systematic Review. Journal of Athletic Training, 47(3), pp.339-357.

6. Akram Pashnameh, Rahim Mirnasouri, Mohammad Nikravan (2014). Relationship between genu valgum, genu varum and flat food Deformities with Static and Dynamic Balance in Female Students of Dorud Islamic Azad University. Asian Journal of Multidisciplinary Studies

7. McCann, R., Kosik, K., Beard, M., Terada, M., Pietrosimone, B. and Gribble, P., 2015. Variations in Star Excursion Balance Test Performance Between High School and Collegiate Football Players. Journal of Strength and Conditioning Research, 29(10), pp.2765-2770.

8. Bhanot, K., Kaur, N., Brody, L., Bridges, J., Berry, D. and Ode, J., 2019. Hip and Trunk Muscle Activity During the Star Excursion Balance Test in Healthy Adults. Journal of Sport Rehabilitation, 28(7), pp.682-691

9. Yun-Won Chae, Ji-Won Park, Seol Park. The Effect of Postural Stability on Genu Varum in Young Adults. The Journal of Korean Society of Physical Therapy 2012;24(6):419-422.

10. Tomas Stølen, Karim Chamari, Carlo Castagna and Ulrik Wisløff 2005. Physiology of Soccer. Sports Medicine, 35(6), pp.501-536

11. Ganavi R. Bow legs and knock knees: is it physiological or pathological? International Journal of Contemporary Pediatrics. 2016;:687-691.

How to cite this article: Shah N, Palkar A. Comparison of dynamic balance in football players with and without genu varum using star excursion balance test. Int J Health Sci Res. 2021; 11(10): 34-40. DOI: https://doi.org/10. 52403/ijhsr.20211006 